\title{
OPERATOR NORMS DETERMINED BY THEIR NUMERICAL RANGES
}

\author{
by COLIN M. MCGREGOR $\dagger$ \\ (Received 19th May 1970)
}

\section{Introduction}

This paper owes its origin to the following question posed by A. M. Sinclair, "If a linear algebra with identity has two equivalent unital algebra norms, $|.|_{1}$ and $|.|_{2}$, whose corresponding numerical radii, $v_{1}$ and $v_{2}$, are equal on the whole algebra, are $\mid . l_{1}$ and $\mid . l_{2}$ related? Are they, for example, necessarily equal?" We do not give a complete answer to this question but are able to give sufficient conditions on algebras of operators for $v_{1}=v_{2}$ to imply $|\cdot|_{1}=|\cdot|_{2}$ That this implication does not hold for an arbitrary algebra with identity is demonstrated by means of a counter-example. The result for operator algebras is used to deduce some essentially non numerical range results for equivalent operator norms.

Given a normed linear space $X$ (over $R$ or $C$ ) we shall write $X^{\prime}$ for the dual space, $X_{1}$ for the closed unit ball of $X$ and $S(X)$ for the set $\{x \in X:\|x\|=1\}$. We shall write $B(X)$ for the algebra of all bounded linear operators on $X$ and 1.| for the operator norm on $B(X)$.

For a detailed account of the theory of numerical ranges see Bonsall and Duncan (1). We present here some of the basic definitions.

Let $A$ be a unital normed algebra with identity $e$. For $a \in A$ the numerical range of $a$ is defined by

$$
V(A, a)=\left\{f(a): f \in S\left(A^{\prime}\right), f(e)=1\right\},
$$

and the numerical radius of $a$ by

$$
v(a)=\sup \{|\lambda|: \lambda \in V(A, a)\} .
$$

Let $X$ be a normed linear space. $B(X)$ is a unital normed algebra with respect to $|$.$| and so each operator T \in B(X)$ has a numerical range and a numerical radius as defined above. There is, however, an alternative definition of the numerical range of an operator. For $T \in B(X)$ the spatial numerical range of $T$ is defined by

$$
V(T)=\left\{f(T x): x \in S(X), f \in S\left(X^{\prime}\right), f(x)=1\right\} .
$$

$\uparrow$ The author wishes to acknowledge the support of a Research Studentship from the Science Research Council. 
It may be shown that the closed convex hull of $V(T)$ is equal to $V(B(X), T)$ and it follows that

$$
\sup \{|\lambda|: \lambda \in V(T)\}=v(T) .
$$

I wish to express my gratitude to my research supervisor, Dr. J. Duncan, for his encouragement and advice during the writing of this paper. I am also indebted to M. J. Crabb for helpful suggestions.

\section{Preliminary results}

Given a convex function $\phi: R \rightarrow R$ we shall write $\phi_{L}^{\prime}$ and $\phi_{R}^{\prime}$ for the left and right derivatives of $\phi$ respectively, these being defined on the whole of $\boldsymbol{R}$.

1. Lemma. Let $\phi: R \rightarrow R$ be convex. Then

$$
(s-t) \phi_{L}^{\prime}(t)+\phi(t) \leqq \phi(s) \quad(s<t) .
$$

Proof. This is routine.

2. Lemma. Let $p$ and $q$ be two norms for $R^{2}$ and $A$ and $B$ the corresponding closed unit balls. If $\xi A \cup \eta B$ is convex for all $\xi, \eta>0$, then there exists $K>0$ such that $p=K q$ on $R^{2}$.

Proof. Suppose that for all $\xi, \eta>0, \xi A \cup \eta B$ is convex. Define $\phi$ and $\psi: R \rightarrow R^{+}$by

$$
\left.\begin{array}{l}
\phi(t)=p(1, t) \\
\psi(t)=q(1, t)
\end{array}\right\} \quad(t \in R) .
$$

Clearly $\phi$ and $\psi$ are continuous and strictly positive. The subadditivity of $p$ and $q$ implies that both $\phi$ and $\psi$ are convex. Let $\xi, \eta>0$. The Minkowski functional for the convex set $\xi A \cup \eta B$ is $\min \{\xi p, \eta q\}$ and the subadditivity of this functional implies that $\min \{\xi \phi, \eta \psi\}$ is convex. We show that for all $t \in R$,

$$
\frac{\phi_{L}^{\prime}(t)}{\phi(t)}=\frac{\psi_{L}^{\prime}(t)}{\psi(t)} \text { and } \frac{\phi_{R}^{\prime}(t)}{\phi(t)}=\frac{\psi_{R}^{\prime}(t)}{\psi(t)}
$$

Suppose that for some $t \in R, \frac{\phi_{L}^{\prime}(t)}{\phi(t)}<\frac{\psi_{L}^{\prime}(t)}{\psi(t)}$.

Let $\psi(t)=\alpha \phi(t)$. Then $\psi_{L}^{\prime}(t)-\alpha \phi_{L}^{\prime}(t)=\varepsilon_{0}$ (say)>0. Choose $\varepsilon_{1} \in\left(0, \frac{1}{4} \varepsilon_{0}\right)$. Then there exists $\delta_{1}>0$ such that $0<h \leqq \delta_{1}$ implies

So in particular,

$$
\left|\psi_{L}^{\prime}(t)-\left\{\frac{\psi(t)-\psi(t-h)}{h}\right\}\right|<\varepsilon_{1} .
$$

$$
\psi\left(t-\delta_{1}\right)<\delta_{1}\left\{\varepsilon_{1}-\psi_{L}^{\prime}(t)\right\}+\psi(t)
$$


Now choose $\varepsilon_{2} \in(0,1)$ such that $\varepsilon_{2} \alpha \phi(t)<\frac{1}{2} \delta_{1} \varepsilon_{0}$ and $\varepsilon_{2}\left|\alpha \phi_{L}^{\prime}(t)\right|<\frac{1}{4} \varepsilon_{0}$. Then we get

$$
\begin{aligned}
\psi\left(t-\delta_{1}\right) & <\delta_{1}\left\{\frac{1}{4} \varepsilon_{0}-\frac{1}{2} \varepsilon_{0}-\varepsilon_{2} \alpha \phi_{L}^{\prime}(t)-\left(1-\varepsilon_{2}\right) \alpha \phi_{L}^{\prime}(t)\right\}-\frac{1}{2} \delta_{1} \varepsilon_{0}+\alpha \phi(t) \\
& <\delta_{1}\left\{-\frac{1}{4} \varepsilon_{0}-\varepsilon_{2} \alpha \phi_{L}^{\prime}(t)\right\}-\delta_{1}\left(1-\varepsilon_{2}\right) \alpha \phi_{L}^{\prime}(t)+\left(1-\varepsilon_{2}\right) \alpha \phi(t) \\
& <-\delta_{1}\left(1-\varepsilon_{2}\right) \alpha \phi_{L}^{\prime}(t)+\left(1-\varepsilon_{2}\right) \alpha \phi(t) \\
& \left.\leqq\left(1-\varepsilon_{2}\right) \alpha \phi\left(t-\delta_{1}\right) \quad \text { (by Lemma } 1\right) .
\end{aligned}
$$

Clearly $\psi(t)>\left(1-\varepsilon_{2}\right) \alpha \phi(t)$. So there exists $\delta_{2} \in\left(0, \delta_{1}\right)$ such that

$$
\psi\left(t-\delta_{2}\right)>\left(1-\varepsilon_{2}\right) \propto \phi\left(t-\delta_{2}\right) \text {. }
$$

We consider the convex function $\min \left\{\left(1-\varepsilon_{2}\right) \alpha \phi, \psi\right\}=\theta$ (say) and obtain a contradiction by showing that

Observe that

$$
\theta\left(t-\delta_{2}\right)>\frac{\delta_{2}}{\delta_{1}} \theta\left(t-\delta_{1}\right)+\frac{\delta_{1}-\delta_{2}}{\delta_{1}} \theta(t)
$$

Thus

$$
\begin{aligned}
\theta\left(t-\delta_{1}\right) & =\psi\left(t-\delta_{1}\right), \\
\theta\left(t-\delta_{2}\right) & =\left(1-\varepsilon_{2}\right) \alpha \phi\left(t-\delta_{2}\right), \\
\theta(t) & =\left(1-\varepsilon_{2}\right) \alpha \phi(t) .
\end{aligned}
$$

$$
\begin{aligned}
& \frac{\delta_{2}}{\delta_{1}} \theta\left(t-\delta_{1}\right)+\frac{\delta_{1}-\delta_{2}}{\delta_{1}} \theta(t)=\frac{\delta_{2}}{\delta_{1}} \psi\left(t-\delta_{1}\right)+\frac{\delta_{1}-\delta_{2}}{\delta_{1}}\left(1-\varepsilon_{2}\right) \alpha \phi(t) \\
& \quad<\frac{\delta_{2}}{\delta_{1}}\left\{-\delta_{1}\left(1-\varepsilon_{2}, \alpha \phi_{L}^{\prime}(t)\right\}+\left(1-\varepsilon_{2}\right) \alpha \phi(t) \quad\right. \text { (by (*) above) } \\
& \left.\quad \leqq\left(1-\varepsilon_{2}\right) \alpha \phi\left(t-\delta_{2}\right) \quad \text { (by Lemma } 1\right) .
\end{aligned}
$$

This contradiction establishes that for all $t \in R$,

$$
\frac{\phi_{L}^{\prime}(t)}{\phi(t)} \geqq \frac{\psi_{L}^{\prime}(t)}{\psi(t)}
$$

It now follows by symmetry, that for all $t \in R$,

$$
\frac{\phi_{L}^{\prime}(t)}{\phi(t)}=\frac{\psi_{L}^{\prime}(t)}{\psi(t)} \text { and } \frac{\phi_{R}^{\prime}(t)}{\phi(t)}=\frac{\psi_{R}^{\prime}(t)}{\psi(t)}
$$

It follows from this that the continuous function $\phi / \psi: R \rightarrow R$ is differentiable on $R$ with derivative zero. Hence there exists $K>0$ such that $\phi=K \psi$ on $R$ and it follows that $p=K q$ on $R^{2}$.

\section{Main results}

Given a normed linear space $X$ we shall say that a subset $A$ of $B(X)$ is an $\alpha$-subset of $B(X)$ if for all $x, y \in S(X)$ and all $\varepsilon>0$ there exists $T \in A$ such that 
$v(T)<1+\varepsilon$ and $\|T x-y\|<\varepsilon$. We shall say that $A$ is an $\alpha_{0}$-subset of $B(X)$ if it is an $\alpha$-subset and $T \in A$ implies that $\lambda T \in A$ for every scalar $\lambda$.

Note that any subset of $B(X)$ which contains all operators of rank 1 is an $\alpha$-subset and $B(X)$ itself is an $\alpha_{0}$-subset.

3. Theorem. Let $\|\cdot\|_{1}$ and $\|\cdot\|_{2}$ be two equivalent norms for a linear space $X$ and let $v_{1}$ and $v_{2}$ be the corresponding numerical radii on $B(X)$. If $v_{1}(T)=v_{2}(T)$ for every operator $T$ in an $\alpha$-subset of $B\left(X,\|.\|_{1}\right)$ then there exists $K>0$ such that $\|\cdot\|_{1}=K\|\cdot\|_{2}$ on $X$.

Proof. Suppose that $A$ is an $\alpha$-subset of $B\left(X,\|\cdot\|_{1}\right)$ and $v_{1}(T)=v_{2}(T)$ for every $T \in A$. Further suppose that there is no $K>0$ such that $\|\cdot\|_{1}=K\left\|_{0}\right\|_{2}$ on $X$. We may assume without loss of generality that there exist $a, b \in X$ such that $\|a\|_{1}<\|a\|_{2}$ and $\|b\|_{1}>\|b\|_{2}$. Write $Y$ for the real linear span of $\{a, b\}$. Let $X^{i}=\left(X,\|\cdot\|_{i}\right)$ and $Y^{i}=\left(Y,\|\cdot\|_{i}\right)$ for $i=1,2$. In view of Lemma 2 we may assume that $Y^{1}{ }_{1} \cup Y^{2}{ }_{1}=W$ (say) is not convex. So there exist $\bar{x}, \bar{y} \in W$ such that the segment $[\bar{x}, \bar{y}]=\{(1-t) \bar{x}+t \bar{y}: 0 \leqq t \leqq 1\} \nsubseteq W$. So $\bar{x}$ and $\bar{y} \in X^{1}{ }_{1} \cup X^{2}{ }_{1}$ and $[\bar{x}, \bar{y}] \notin X^{1}{ }_{1} \cup X^{2}{ }_{1}$. Observe that $\bar{x}, \bar{y}$ and 0 are not collinear. Now there exist $z^{\prime}$ and $z$ such that $z^{\prime}$ lies in the open segment

$$
(\bar{x}, \bar{y})=\{(1-t) \bar{x}+t \bar{y}: 0<t<1\}, \quad z \in\left(0, z^{\prime}\right) \text { and }\|z\|_{1}=\|z\|_{2}=1 .
$$

Let

where

$$
x=\left(1-t_{0}\right) \bar{x}+t_{0} z^{\prime},
$$

$$
t_{0}=\sup \left\{t \in[0,1):\left\|(1-t) \bar{x}+t z^{\prime}\right\|_{1} \leqq 1\right\}
$$

and $y=\left(1-s_{0}\right) \bar{y}+s_{0} z^{\prime}$, where

$$
s_{0}=\sup \left\{t \in[0,1):\left\|(1-t) \bar{y}+t z^{\prime}\right\|_{2} \leqq 1\right\} .
$$

Clearly $x \in\left[\bar{x}, z^{\prime}\right) \cap S\left(X^{1}\right)$ and $y \in\left[\bar{y}, z^{\prime}\right) \cap S\left(X^{2}\right)$. Now there exists

$$
w \in\left(0, \frac{1}{2}\left(x+z^{\prime}\right)\right)
$$

such that $\|w\|_{1}=1<\|w\|_{2}$. Let $\left\{w^{\prime}\right\}=(w, y) \cap\left(0, z^{\prime}\right)$. We may choose $u^{\prime} \in\left(w^{\prime}, y\right)$ with $\left\|u^{\prime}\right\|_{2}>1$. Then there exists $u \in\left(0, u^{\prime}\right)$ such that

$$
\|u\|_{2}=1<\|u\|_{1} \text {. }
$$

Let $f \in X^{\prime}$ with $\|f\|_{2}=1=f(u)$. Then choose $\varepsilon$ such that

$$
0<\varepsilon<\frac{\|u\|_{1}-1}{1+\|f\|_{1}\|u\|_{1}} .
$$

Then $1+\varepsilon<\|u\|_{1}\left(1-\|f\|_{1} \varepsilon\right)$. Since $A$ is an $\alpha$-subset of $B\left(X,\|\cdot\|_{1}\right)$, there exists $T \in A$ such that $v_{1}(T)<1+\varepsilon$ and $\left\|T\left(u /\|u\|_{1}\right)-w\right\|_{1}<\varepsilon$. However,

$$
\begin{aligned}
1=f(u)<f\left(u^{\prime}\right) \leqq & (1-t)|f(w)|+t|f(y)| \\
& (\text { for some } t \in(0,1)) \\
\leqq & (1-t)|f(w)|+t
\end{aligned}
$$


So $|f(w)|>1$ and so

$$
\begin{aligned}
|f(T u)| & \geqq\left|f\left(\|u\|_{1} w\right)\right|-\left|f\left(T u-\|u\|_{1} w\right)\right| \\
& >\|u\|_{1}-\|f\|_{1}\|u\|_{1} \varepsilon \\
& >1+\varepsilon .
\end{aligned}
$$

Thus $v_{1}(T)<1+\varepsilon<v_{2}(T)$ and this contradiction establishes the result.

4. Theorem. Let $\|\cdot\|_{1}$ and $\|\cdot\|_{2}$ be two equivalent norms for a linear space $X$ and let $U$ be a relative neighbourhood of 0 in some $\alpha_{0}$-subset of $B\left(X,\|\cdot\|_{1}\right)$. If the operator norms corresponding to $\|\cdot\|_{1}$ and $\|\cdot\|_{2}$ agree on $I+U$ then they agree on the whole of $B(X)$ and there exists $K>0$ such that $\|\cdot\|_{1}=K\|\cdot\|_{2}$ on $X$.

Proof. This follows from Theorem 3 using the fact (see Bonsall and Duncan (1), $\$ 2$, Theorem 5) that for each $T \in B(X)$ there exists a scalar $\lambda$ with modulus 1 such that

$$
v_{i}(T)=\lim _{t \rightarrow 0+} \frac{|I+t \lambda T|_{i}-1}{t} \quad(i=1,2)
$$

(where subscripts $i$ denote correspondence with $\|\cdot\|_{i}$ ).

5. Corollary. With the notation of Theorem 4, if $|T|_{1}=|T|_{2}$ for every invertible operator $T$ in $B(X)$ then $|.|_{1}$ and $|.|_{2}$ agree on the whole of $B(X)$.

6. Theorem. Let $\|\cdot\|_{1}$ and $\left\|_{1}\right\|_{2}$ be two equivalent Banach space norms for a linear space $X$ and let $U$ be a relative neighbourhood of 0 in some $\alpha_{0}$-subset of $B\left(X,\|\cdot\|_{1}\right)$. If the operator norms corresponding to $\|\cdot\|_{1}$ and $\|\cdot\|_{2}$ agree on $\exp (U)$ then they agree on the whole of $B(X)$.

Proof. This follows from Theorem 3 using the fact (see Bonsall and Duncan (1), $\S 3$, Theorem 4) that for each $T \in B(X)$ there exists a scalar $\lambda$ with modulus 1 such that

$$
v_{i}(T)=\lim _{t \rightarrow 0+} \frac{1}{t} \log |\exp (t \lambda T)|_{i} \quad(i=1,2)
$$

(where subscripts $i$ denote correspondence with $\|\cdot\|_{i}$ ).

\section{An example}

We give an example of an algebra with identity, having two different unital Banach algebra norms for which the corresponding numerical ranges are identical.

We define two Banach space norms for $C^{2}$ as follows: for $(x, y) \in C^{2}$

$$
\begin{aligned}
& \|(x, y)\|_{1}= \begin{cases}|x| \exp \left(\frac{1}{2}|y / x| \log 2\right) & \text { if } x \neq 0 \text { and }|y / x| \leqq 2 \\
|y| & \text { otherwise }\end{cases} \\
& \|(x, y)\|_{2}=\left(|x|^{2}+|y|^{2}\right)^{\frac{1}{2}} .
\end{aligned}
$$

Subscripts 1 and 2 shall denote correspondence with $\|\cdot\|_{1}$ and $\|\cdot\|_{2}$ respectively. 
Let $S$ be the unilateral shift operator on $C^{2}$ given by

$$
S(x, y)=(0, x) \quad\left((x, y) \in C^{2}\right) .
$$

It may be verified (see (2)) that $V_{1}(S)=V_{2}(S)=\left\{\lambda \in C:|\lambda| \leqq \frac{1}{2}\right\}$.

Let $A$ be the subalgebra of $B\left(C^{2}\right)$ generated by $I$ and $S$. Then

$$
A=\{\xi I+\eta S: \xi, \eta \in C\} .
$$

It follows that for all $T \in A, V_{1}(T)=V_{2}(T)$. We show, however, that

$$
|I+S|_{1}<1 \cdot 6<|I+S|_{2} \text {. }
$$

For $(x, y) \in C^{2},(I+S)(x, y)=(x, x+y)$. We show first that for

$$
\|(x, y)\|_{1}=1, \quad\|(x, x+y)\|_{1}<1 \cdot 55 .
$$

Let $(x, y) \in C^{2}$ with $\|(x, y)\|_{1}=1$.

(a) If $x=0$ then $\|(x, x+y)\|_{1}=\|(x, y)\|_{1}<1.55$.

(b) If $x \neq 0,|y / x|>2$ and $|(x+y) / x|>2$ then $|y|=1$ and

$$
\begin{aligned}
\|(x, x+y)\|_{1} & =|x+y| \\
& <\frac{1}{2}|y|+|y|<1.55 .
\end{aligned}
$$

(c) If $x \neq 0,|y / x|>2$ and $|(x+y) / x| \leqq 2$ then $|y|=1$ and

$$
\begin{aligned}
\|(x, x+y)\|_{1} & =|x| \exp \left(\frac{1}{2}|(x+y) / x| \log 2\right) \\
& \leqq|x| \exp (\log 2)<1.55 .
\end{aligned}
$$

(d) If $x \neq 0,|y / x| \leqq 2$ and $|(x+y) / x|>2$ then

$$
|x| \exp \left(\frac{1}{2}|y / x| \log 2\right)=1 \text { and }\|(x, x+y)\|_{1}=|x+y| .
$$

Let $\beta=|y| x \mid$. So $|x|=\exp \left(-\frac{1}{2} \beta \log 2\right)$ and

$$
\|(x, x+y)\|_{1} \leqq|x|+|y|=(1+\beta) \exp \left(-\frac{1}{2} \beta \log 2\right)
$$

and from this it is routine to verify that $\|(x, x+y)\|_{1}<1.55$.

(e) If $x \neq 0,|y / x| \leqq 2$ and $1(x+y) / x \mid \leqq 2$ then and

$$
|x| \exp \left(\frac{1}{2}|y| x \mid \log 2\right)=1
$$

$$
\begin{aligned}
\|(x, x+y)\|_{1} & =|x| \exp \left(\frac{1}{2}|(x+y) / x| \log 2\right) \\
& \leqq \exp \left(-\frac{1}{2}|y / x| \log 2\right) \exp \left(\frac{1}{2}(1+|y / x|) \log 2\right) \\
& <1 \cdot 55 .
\end{aligned}
$$

It follows from (a), (b), (c), (d) and (e) that $|I+S|_{1}<1 \cdot 6$.

Now consider $(a, b)=(2, \sqrt{5}-1)$. It is easily verified that

and hence $|I+S|_{2}>1 \cdot 6$.

$$
\frac{\|(a, a+b)\|_{2}}{\|(a, b)\|_{2}}>1.6
$$




\section{REFERENCES}

(1) F. F. BONSALL and J. DUNCAN, Numerical ranges of operators on normed spaces and of elements of normed algebras, London Math. Soc. Lecture Note Series, No. 2 (1971).

(2) J. Duncan, C. M. McGregor, J. D. Pryce and A. J. White, The numerical index of a normed space, J. London Math. Soc. (2) 2 (1970), 481-488.

Department of Mathematics

King's College, AberdeEN 